\title{
Are there socioeconomic inequalities in cardiovascular risk factors in childhood, and are they mediated by adiposity? Findings from a prospective cohort study
}

\author{
LD Howe $^{1,2}$, B Galobardes ${ }^{1}$, N Sattar ${ }^{3}$, AD Hingorani ${ }^{4}$, J Deanfield $^{5}$, AR Ness ${ }^{6}$, G Davey-Smith ${ }^{1,2}$ and \\ DA Lawlor ${ }^{1,2}$
}

${ }^{1}$ Department of Social Medicine, University of Bristol, Bristol, UK; ${ }^{2}$ Department of Social Medicine, MRC Centre for Causal Analyses in Translational Epidemiology, University of Bristol, Bristol, UK; ${ }^{3}$ BHF Glasgow Cardiovascular Research Centre, Faculty of Medicine, University of Glasgow, Glasgow, Scotland, UK; ${ }^{4}$ Epidemiology and Public Health, University College London, London, UK; ${ }^{5}$ Institute of Child Health, University College London, London, UK and ${ }^{6}$ Department of Oral and Dental Science, University of Bristol, Bristol, UK

Background: Socioeconomic gradients in adiposity were not present during childhood for previous generations, but have emerged in contemporary children. It is unknown whether this translates to socioeconomic gradients in associated cardiovascular risk factors in children, with consequent implications for inequalities in coronary heart disease (CHD) and diabetes when these children reach adulthood.

Methods: Using data from 7772 participants aged 10-years from the Avon Longitudinal Study of Parents and Children, we examined the association between maternal education and a large number of cardiovascular risk factors (cholesterol, triglycerides, high-density lipoprotein, apolipoprotein, adiponectin, leptin, C-reactive protein (CRP), interleukin-6 (IL-6) and systolic and diastolic blood pressure), and examined whether inequalities were mediated by adiposity, measured by dual energy X-ray absorptiometry (DXA)-assessed total fat mass.

Results: There were socioeconomic differences in a number of the cardiovascular risk factors (apolipoprotein B, systolic and diastolic blood pressure, CRP, leptin and IL-6). Inequalities were greater in girls than boys. Inequalities in CRP and leptin were completely mediated by adiposity. Inequalities in other cardiovascular risk factors were partially mediated by adiposity.

Conclusion: This study showed important socioeconomic inequalities in adiposity and associated cardiovascular risk factors in a contemporary UK population of 10-year-old children. Differences between contemporary children and previous generations in the socioeconomic patterning of cardiovascular risk factors suggest future adults may have greater inequalities in diabetes and CHD than current adults. These findings highlight the importance of interventions aimed at preventing obesity in childhood, particularly among those of lower socioeconomic position.

International Journal of Obesity (2010) 34, 1149-1159; doi:10.1038/ijo.2010.52; published online 16 March 2010

Keywords: ALSPAC; child; inequality; cardiovascular

\section{Background}

Low socioeconomic position (SEP) in childhood is associated with higher risk of coronary heart disease $(\mathrm{CHD})^{1-3}$ and diabetes ${ }^{4,5}$ in later life, but the mechanisms underlying this are unclear. One possibility is that children from more deprived backgrounds are likely to be more obese and

Correspondence: Dr LD Howe, Department of Social Medicine, University of Bristol, Oakfield House, Oakfield Grove, Bristol BS8 2BN, UK.

E-mail: laura.howe@bristol.ac.uk

Received 3 November 2009; revised 21 January 2010; accepted 31 January 2010; published online 16 March 2010 childhood obesity mediates the association of childhood SEP with CHD in adulthood. A systematic review of studies in contemporary populations of children from high-income countries found that most studies show socioeconomic differentials in adiposity, with those from more deprived socioeconomic backgrounds being more adipose. ${ }^{6}$ Furthermore, greater body mass index (BMI) and obesity in childhood from age 7 onwards to early adulthood have been shown to be associated with CHD risk in adulthood. ${ }^{7}$ However, it is unknown whether this association is due to tracking of BMI from childhood to adulthood or whether permanent changes in cardiovascular risk factors, such as lipid and blood pressure levels, occur in childhood as a result 
of greater adiposity. If the latter is true these adiposityrelated risk factors changes might mediate the association of childhood SEP with adult CHD. As well as being associated with future risk of $\mathrm{CHD}$, individuals from poorer socioeconomic backgrounds in childhood have been found to be more obese, more dyslipidaemic and more insulin resistant in adulthood than those from higher socioeconomic groups, ${ }^{8,9}$ but few studies have been able to examine whether childhood SEP is associated with variation in vascular and metabolic traits in childhood, and if so to what extent these associations are mediated by adiposity.

One study in Denmark $(n=933)$, Estonia $(n=1103)$ and Portugal $(n=1153)$ showed socioeconomic differentials in BMI, waist circumference, lipids (high-density lipoprotein (HDL), low-density lipoprotein and triglycerides) and circulating insulin. ${ }^{10}$ In Estonia and Portugal (relatively low-income countries), the socioeconomic differential in BMI was the opposite (lower BMI and waist circumference in those from poorer and less well-educated families) of that observed in Denmark, a high-income country, where findings were consistent with results from other high-income countries, ${ }^{6}$ that is, BMI and waist circumference were higher in those from poorer and less well-educated families. The socioeconomic gradients in metabolic markers were in the directions that would be anticipated given the observed relationships between SEP and BMI, that is, levels of low-density lipoprotein, triglycerides and circulating insulin were higher among those of lower SEP in Denmark and lower among those of lower SEP in Estonia and Portugal, and vice versa for HDL. Data from the 1992-2002 National Health and Nutrition Examination Survey from the United States showed that neither income nor education were associated with the metabolic syndrome in adolescents (based on having three or more extreme values for waist circumference, blood pressure, triglycerides, or fasting glucose, using national reference data). ${ }^{11}$ A review of studies published up to the year 2000 found little socioeconomic patterning of blood pressure, cholesterol, C-reactive protein (CRP), homocysteine or fibrinogen during childhood or early adulthood, but most studies were small. ${ }^{12}$ A study in Northern Ireland ( $N=509)$ found little socioeconomic difference in biological risk factors for $\mathrm{CHD}$ at ages 12 or 15, but did find differences in behaviours such as physical activity, diet and smoking, leading to the suggestion that socioeconomic differences in risky behaviours are likely to emerge earlier than differences in physiological factors. ${ }^{13}$

In this study, we analyse data from a research clinic of the Avon Longitudinal Study of Parents and Children (ALSPAC) held when the children were approximately 10-years old (mean age 9.9 years). A previous publication from this study showed that children from lower socioeconomic backgrounds tend to have higher total fat mass and greater BMI, but that there was no social gradient in lean mass. ${ }^{14}$ The investigators also showed that inequalities in adiposity were wider when using DXA-assessed total fat mass compared with BMI, and concluded that using BMI as a measure of adiposity in children may underestimate inequalities. We extend this earlier work by examining socioeconomic differentials in cardiovascular risk factors, and the extent to which these associations are mediated by adiposity measured by DXA-assessed total fat mass.

\section{Materials and methods}

\section{Study population}

ALSPAC is a prospective cohort study investigating the health and development of children. The full study methodology has been published elsewhere, ${ }^{15}$ and is also detailed on the study website (www.bristol.ac.uk/alspac). Briefly, pregnant women resident in one of three Bristolbased health districts with an expected date of delivery between 1 April 1991 and 31 December 1992 were invited to take part in the study. Of these women, 14541 were recruited. From these pregnancies, there were 14062 liveborn children, 13988 of whom were alive at 1 year. This study uses data from a clinic held at 10 years of age (clinics held between January 2001 and January 2003, mean age of children at clinic visits 9.9 years, s.d. 0.3), to which all surviving children with known contact details and whose parents had consented to continued participation were invited. A total of 7722 children attended the clinic, approximately $65 \%$ of those invited. Ethical approval for the study was obtained from the ALSPAC Law and Ethics Committee and the Local Research Ethics Committees.

\section{Measurements}

A questionnaire at 32 weeks gestation asked mothers to report their educational attainment. Maternal educational attainment was categorised as below O-level (ordinary level; exams taken in different subjects usually at age 15-16 at the completion of legally required school attendance, equivalent to today's General Certificate of Secondary Education), O-level only, A-level (advanced-level; exams taken in different subjects usually at age 18), or university degree or above.

Puberty stage was assessed using questionnaires containing line drawings and questions based on stages described by Tanner, ${ }^{16,17}$ which were completed within 1 year of attendance at the clinic at age 10; Tanner stage ranges between I and V, with I being least sexually mature.

All other variables used in this analysis were from the clinic at age 10. Adiposity was measured by DXA-assessed fat mass. A Lunar prodigy narrow fan beam densitometer was used to perform a whole body DXA scan in which bone content, lean and fat masses are measured. Total fat mass $(\mathrm{kg})$ is used in these analyses. Height was measured to the last complete millimetre ( $\mathrm{mm}$ ) using the Harpenden Stadiometer. Blood pressure was measured using a Dinamap 9301 Vital Signs Monitor (Critikon, Tampa, FL, USA). Two readings of systolic and diastolic blood pressure were recorded and the mean of each has been calculated. Non-fasting blood 
samples were taken using standard procedures with samples immediately spun and frozen at $-80^{\circ} \mathrm{C}$. The measurements were assayed in 2008 after a median of 7.5 years in storage with no previous freeze-thaw cycles during this period. Plasma lipids (total cholesterol, triglycerides and HDL-C) were performed by modification of the standard Lipid Research Clinics Protocol using enzymatic reagents for lipid determinations. $^{18}$ Apo A1 and Apo B were measured by immunoturbidimetric assays (Roche UK, Welwyn Garden City, UK). Leptin was measured by an in house enzymelinked immunosorbent assay validated against commercial methods. ${ }^{19}$ Adiponectin and high-sensitivity interleukin-6 (IL-6) were measured by enzyme-linked immunosorbent assay (R\&D systems, Abingdon, UK) and CRP was measured by automated particle-enhanced immunoturbidimetric assay (Roche UK). All assay coefficients of variation were $<5 \%$. CRP, leptin, IL-6 and DXA-assessed total fat mass were all right-skewed, so log transformations were used in all analyses.

\section{Statistical analysis}

The initial steps in our analyses were to quantify the associations between (i) maternal education and DXAassessed total fat mass, (ii) maternal education and each of the cardiovascular risk factors and (iii) DXA-assessed total fat mass and each of the cardiovascular risk factors. Inequalities in (i) DXA-assessed total fat mass and (ii) each cardiovascular risk factor were summarised using the slope index of inequality (SII). The SII assumes an underlying continuous distribution of SEP; it is interpreted as the mean difference comparing the individual of lowest SEP with the individual of highest SEP on this hypothetical underlying continuous scale. ${ }^{20}$ When calculating the SII for DXA-assessed fat mass, we adjusted analyses for age, height and height squared. Adjustment for age was carried out in calculating the SIIs for cardiovascular risk factors. Gender differences in these inequalities were examined by testing for interactions. To examine whether any observed gender differences in adiposity inequalities were driven by differences in biological age between girls and boys, we tested for interactions with age and determined the level of adiposity inequality across tertiles of age. To quantify the associations between DXAassessed total fat mass and each of the cardiovascular risk factors, we used linear regression adjusted for age, height and height squared. Again, we examined gender differences by testing for interactions.

We then examined the degree to which inequalities in the cardiovascular risk factors were mediated by adiposity. For one variable to mediate the association of an exposure with an outcome, it has to fulfil the following criteria: (i) be causally influenced by the exposure of interest, (ii) causally influence the outcome and (iii) attenuate the association of the exposure with the outcome. ${ }^{21,22}$ For our specific question of whether adiposity mediated the association of SEP with cardiovascular risk factors, we are assuming that SEP is causally related to adiposity and that adiposity is causally related to cardiovascular risk factors. In prospective cohort studies in adults, there are robust associations between adiposity and cardiovascular risk factors in a wide range of populations, and in randomised controlled trials of interventions that effectively reduce weight these risk factors are reduced in ways predicted from observational studies. ${ }^{23,24}$ In children there are fewer such trials but evidence here also suggests that weight reduction results in expected improvements in cardiovascular risk factors. ${ }^{25,26}$ Furthermore, Mendelian randomisation studies, in which genetic variants that are robustly associated with adiposity throughout life are used as instrumental variables, support a causal effect of greater adiposity on adverse cardiovascular risk factors. ${ }^{27,28}$ It is less easy to provide evidence that SEP causally affects childhood adiposity, although the association is consistently observed across most high-income countries. ${ }^{6}$

Traditionally, mediation has been assessed by standard regression techniques; mediation by adiposity is assumed to be present if the association between SEP and a cardiovascular risk factor is attenuated to the null after adjustment for adiposity. ${ }^{29}$ In our analyses, we used path analysis ${ }^{30}$ to examine mediation of the inequalities in cardiovascular risk factors by adiposity. Path analysis is preferable to standard regression techniques because it allows estimation of the total effect of maternal education on each cardiovascular risk factor (equivalent to the crude, unadjusted association) and the indirect effect of maternal education (that is, the effect mediated by adiposity), with confidence intervals around each of these estimates. ${ }^{31}$ In standard regression methods, the 'adjusted' effect estimate is a measure of the association between SEP and the cardiovascular risk factor holding adiposity constant. In contrast, the indirect effect estimate from path analysis holds SEP constant. Indirect effects therefore reflect the difference in cardiovascular risk factor that would be observed if the whole population moved from the adiposity level of the highest SEP group to the adiposity level of the lowest SEP group.

We present standardised linear regression coefficients for both the total and indirect effects of maternal education on each cardiovascular risk factor, that is, each coefficient for total effects represents the s.d. change in the cardiovascular risk factor for a one s.d. increase in maternal education. The size of these standardised total and indirect coefficients can then be compared with estimate the proportion of the SEP-cardiovascular association that is mediated by adiposity. For example, if the total coefficient was 1 and the indirect coefficient was 0.5 , we could estimate that half of the effect of maternal education on the cardiovascular risk factor was operating through adiposity. Analyses were adjusted for age, height and height squared.

Path analyses were conducted in MPlus version 5.21 (Muthén and Muthén, Los Angeles, CA, USA), ${ }^{32}$ other analyses were conducted using Stata version 10.1 (Stata Corporation, Texas 2008, College Station, TX, USA). 
Dealing with missing data

Of the 7722 children attending the clinic, $8.8 \%$ had missing data for maternal education and 5.0\% for DXA-assessed total fat mass. Levels of missing data were $1.5 \%$ for blood pressure. Missing data levels are higher for the blood assay, 34.2\%, because some parents and children did not consent to the blood test.

Missing data levels were higher among children who were female, older, more adipose, and who had lower maternal and paternal education, lower parity mothers, lower birth weight and length, lower maternal age, lower gestational age at delivery, shorter and heavier mothers. We used multivariate multiple imputation to impute missing variables, including all covariables and potential predictors in the imputation equations. We used switching regression in Stata as described by Royston. ${ }^{33}$ We carried out 20 cycles of regression switching and generated 10 imputation data sets. The multiple multivariate imputation approach creates a number of copies of the data (in this case we generated 10 copies) each of which has imputed values for those that are missing, with an appropriate level of randomness, by chained equations. ${ }^{33}$ The main analysis results are obtained by averaging across the results from each of these 10 data sets using Rubin's rules and the procedure takes account of uncertainty in the imputation as well as uncertainty because of random variation. ${ }^{33}$ We imputed data for those with missing data who attended the 10-year follow-up clinic. Hence, the analyses based on these multivariate imputations all include data from 7722 participants.

We repeated all analyses including only those with complete data (without imputation). Multiple imputation resulted in some strengthening of associations between SEP and both adiposity and vascular and metabolic markers, because those with missing data are more likely to be of lower SEP and more adipose. Differences between analysis of the non-missing data set and the imputed data sets were, however, small. Multiple imputation results are presented in this paper, and non-missing data set analysis is available in web tables.

\section{Additional indicators of SEP and adiposity}

We repeated analyses using paternal education and household social class as indicators of SEP. Results were very similar to those presented here for maternal education and so are not presented or discussed further (available on request from investigators). We also repeated analyses using BMI and waist circumference as measures of adiposity. Consistent with findings from a previous study using these data, ${ }^{14}$ observed associations between adiposity and both SEP and the cardiovascular risk factors were somewhat stronger when DXA-assessed fat mass was used compared with BMI. Results using waist circumference as the adiposity measure tended to be intermediate to those using BMI and DXA-assessed fat mass (available on request from investigators).

\section{Results}

Children attending the 10-year follow-up clinic tended to have better educated mothers, older mothers and higher birth weights compared with the full cohort, but no differences in maternal pre-pregnancy BMI were observed (Supplementary table 1).

The socioeconomic profile of clinic participants and the mean levels of DXA-assessed fat mass and each of the cardiovascular risk factors are presented in Table 1. Girls tended to be more adipose than boys and have higher levels of the cardiovascular risk factors, for example, mean CRP levels were $0.22 \mathrm{mgl}^{-1}$ in boys and $0.34 \mathrm{mgl}^{-1}$ in girls. Information on pubertal status was available in $88 \%$ of children attending the research clinic. Most were at an early Tanner stage ( $82 \%$ stages I or II; $96 \%$ stages I-III) and so it is unlikely that associations will have been affected by puberty.

\section{Association of maternal education with adiposity}

The relationship between DXA-assessed total fat mass and SEP differed between boys and girls ( $P$-value for interaction 0.0052). All analyses are therefore presented separately for boys and girls.

Both boys and girls showed socioeconomic differentials in DXA-assessed total fat mass, with children from lower SEP households being more adipose (Table 2 and Supplementary table 2 for analysis of non-missing data set). Inequalities were wider in girls compared with boys.

When we looked at associations by categories of the SEP indicators (as opposed to calculating the SII as a summary measure) it appeared that not all associations were linear across the distribution (Figure 1). Among girls, there was a downward trend in DXA-assessed total fat mass across increasing categories of maternal education. In boys, however, there appeared to be more of a threshold effect, socioeconomic differences in DXA-assessed total fat mass were largely driven by lower levels among children whose mothers hold a degree and little socioeconomic gradient across the lower categories of maternal education (mean levels of DXA-assessed fat mass across categories of maternal education are presented in Supplementary table 3).

To examine whether the difference in adiposity inequalities between boys and girls was driven by the girls being biologically older (that is, closer to puberty), we determined how the inequalities changed with increasing age. Within both boys and girls, there was evidence of a gradient in inequality across age $(P$-value for interaction between age and maternal education $<0.001$ for both boys and girls). When the analyses are stratified by tertiles of age, however, the trend of widening inequality with increasing age was observed clearly within girls but not within boys (Figure 2). 
Table 1 Characteristics of study participants

\begin{tabular}{|c|c|c|}
\hline \multicolumn{3}{|c|}{ Parental socioeconomic characteristics ${ }^{a}$} \\
\hline \multirow[t]{4}{*}{ Maternal education } & Less than O-level & $1577(22.4$ \\
\hline & O-level & $2476(35.2$ \\
\hline & A-level & $1875(26.6$ \\
\hline & Degree or above & 1117 (15.9 \\
\hline
\end{tabular}

Offspring adiposity

\begin{tabular}{|c|c|c|c|c|}
\hline & & oys & & Girls \\
\hline & $\mathrm{N}$ & Mean $(95 \% \mathrm{Cl})$ & $\mathrm{N}$ & Mean $(95 \% \mathrm{Cl})$ \\
\hline Total fat mass $(\mathrm{kg})^{\mathrm{b}}$ & 3617 & $6128.88(6009.95-6250.17)$ & 3716 & $8558.06(8418.56-8699.87)$ \\
\hline
\end{tabular}

Offspring levels of cardiovascular risk factor

\begin{tabular}{|c|c|c|c|c|}
\hline & \multicolumn{2}{|c|}{ Boys } & \multicolumn{2}{|c|}{ Girls } \\
\hline & $\mathrm{N}$ & Mean $(95 \% \mathrm{Cl})$ & $\mathrm{N}$ & Mean $(95 \% \mathrm{Cl})$ \\
\hline Cholesterol $\left(\mathrm{mmoll}^{-1}\right)$ & 2575 & $4.20(4.18-4.23)$ & 2511 & $4.34(4.31-4.37)$ \\
\hline Triglycerides $\left(\mathrm{mmoll}^{-1}\right)$ & 2575 & $1.11(1.09-1.13)$ & 2511 & $1.17(1.15-1.19)$ \\
\hline High-density lipoprotein $\left(\mathrm{mmoll}^{-1}\right)$ & 2575 & $1.44(1.42-1.45)$ & 2511 & $1.37(1.35-1.38)$ \\
\hline Apolipoprotein A1 (mg per 100 ml) & 2575 & $138.40(137.61-139.18)$ & 2511 & $133.70(132.93-134.47)$ \\
\hline Apolipoprotein B (mg per 100 ml) & 2573 & $57.00(56.50-57.47)$ & 2511 & $61.49(60.96-62.02)$ \\
\hline Adiponectin $\left(\mathrm{mg} \mathrm{ml}^{-1}\right)$ & 2575 & $12.74(12.53-12.94)$ & 2510 & $13.53(13.31-13.75)$ \\
\hline C-reactive protein $\left(\mathrm{mgl}^{-1}\right)^{\mathrm{b}}$ & 2575 & $0.22(0.21-0.23)$ & 2511 & $0.34(0.33-0.36)$ \\
\hline Leptin $\left(n \mathrm{~g} \mathrm{ml}^{-1}\right)^{\mathrm{b}}$ & 2575 & $4.77(4.64-4.92)$ & 2509 & $7.58(7.36-7.80)$ \\
\hline Interleukin-6 $\left(\mathrm{pg} \mathrm{m}^{-1}\right)^{\mathrm{b}}$ & 2567 & $0.77(0.74-0.79)$ & 2509 & $0.96(0.93-0.99)$ \\
\hline Systolic blood pressure $(\mathrm{mm} \mathrm{Hg})$ & 3750 & $102.52(102.23-102.81)$ & 3857 & $103.09(102.78-103.39)$ \\
\hline Diastolic blood pressure $(\mathrm{mm} \mathrm{Hg})$ & 3750 & $57.19(56.98-57.39)$ & 3860 & $57.75(57.55-57.95)$ \\
\hline
\end{tabular}

Pubertal stage assessed by Tanner questionnaires

Boys

Prevalence, $\mathrm{n} / \mathrm{N}(\%)$
Girls

Prevalence, $\mathrm{n} / \mathrm{N}(\%)$

\begin{tabular}{lcc}
\hline I & $1300 / 3223(40.3)$ & $2458 / 3555(69.1)$ \\
II & $998 / 3223(31.0)$ & $798 / 3555(22.5)$ \\
III & $704 / 3223(21.8)$ & $242 / 3555(6.8)$ \\
IV & $215 / 3223(6.7)$ & $56 / 3555(1.6)$ \\
V & $6 / 3223(0.2)$ & $1 / 3555(0.03)$ \\
\hline
\end{tabular}

Abbreviation: $\mathrm{Cl}$, confidence interval. ${ }^{\mathrm{a} D}$ Distributions do not differ by gender and are presented for male and female offspring combined. ${ }^{\mathrm{b}} \mathrm{Geometric}$ means.

\section{Association of maternal education with cardiovascular risk factors}

Evidence for gender differences in the relationship between SEP and systolic and diastolic blood pressure was not strong ( $P$-values for gender interactions 0.13. and 0.02). There was strong evidence of gender differences in the associations of maternal education with all other risk factors ( $P$-values for interactions all $\leqslant 0.001)$. All results were therefore presented separately for boys and girls, including blood pressure analyses for consistency.

As with adiposity, associations between SEP and cardiovascular risk factors tended to be stronger among girls than boys. For both boys and girls, weak associations were observed between maternal education and CRP, IL-6 and leptin, and stronger associations were observed for Apo B, systolic and diastolic blood pressure (diastolic blood pressure girls only). Children of less educated mothers had higher values of each of these risk factors (Table 2, Supplementary table 2 for analysis of non-missing data set, and Supplementary table 4 for mean levels across categories of maternal education). There was little evidence of socioeconomic differentials in the other cardiovascular risk factors (cholesterol, triglycerides, HDL, Apo A1 and adiponectin).

Association of adiposity with cardiovascular risk factors There was evidence of gender differences in the associations of some but not all cardiovascular risk factors with DXAassessed total fat mass (for example, $P$-values for interactions 0.003 for triglycerides, $<0.001$ for adiponectin, $<0.001$ for leptin, 0.41 for systolic blood pressure and 0.27 for IL-6). However, for clarity and consistency all results are presented separately for boys and girls.

There was evidence for an association between DXAassessed total fat mass and each of the cardiovascular risk factors (Table 3, and Supplementary table 5 for analysis of 
Table 2 Inequalities in DXA-assessed total fat mass and cardiovascular risk factors at 9.9 years, quantified by the SII by maternal education.

\begin{tabular}{|c|c|c|c|c|}
\hline & \multicolumn{2}{|c|}{ Boys, $\mathrm{N}=3809$} & \multicolumn{2}{|c|}{ Girls, $N=3913$} \\
\hline & SII & $(95 \% \mathrm{Cl})$ & SII & $(95 \% \mathrm{Cl})$ \\
\hline \multicolumn{5}{|c|}{ Inequalities in adiposity } \\
\hline \multicolumn{5}{|c|}{ difference in geometric means comparing most to least deprived (null value $=1$ ) } \\
\hline Total fat mass ${ }^{a}$ & 1.21 & $(1.08$ to 1.36$)$ & 1.34 & (1.23 to 1.46$)$ \\
\hline \multicolumn{5}{|c|}{ Inequalities in cardiovascular risk factors } \\
\hline \multicolumn{5}{|c|}{ difference in means comparing most to least deprived (null value =0) } \\
\hline Cholesterol $\left(\mathrm{mmoll}^{-1}\right)$ & 0.094 & $(-0.0059$ to 0.19$)$ & 0.098 & $(-0.017$ to 0.21$)$ \\
\hline Triglycerides $\left(\mathrm{mmoll}^{-1}\right)$ & 0.0016 & $(-0.088$ to 0.091$)$ & 0.011 & $(-0.092$ to 0.11$)$ \\
\hline High-density lipoprotein $\left(\mathrm{mmoll}{ }^{-1}\right)$ & 0.017 & $(-0.027$ to 0.061$)$ & -0.021 & $(-0.066$ to 0.024$)$ \\
\hline Apolipoprotein A1 (mg per $100 \mathrm{ml}$ ) & 2.20 & $(-0.66$ to 5.05$)$ & 0.60 & $(-2.50$ to 3.70$)$ \\
\hline Apolipoprotein B (mg per $100 \mathrm{ml}$ ) & 2.57 & (0.88 to 4.26$)$ & 3.53 & (1.55 to 5.52$)$ \\
\hline Adiponectin $\left(\mathrm{mg} \mathrm{ml}^{-1}\right)$ & 0.41 & $(-0.34$ to 1.16$)$ & -0.44 & $(-1.35$ to 0.47$)$ \\
\hline Systolic blood pressure & 2.63 & (1.52 to 3.73$)$ & 2.82 & (1.69 to 3.94$)$ \\
\hline Diastolic blood pressure & 1.25 & (0.45 to 2.04$)$ & 1.73 & (0.97 to 2.49$)$ \\
\hline \multicolumn{5}{|c|}{ difference in geometric means comparing most to least deprived (null value $=1$ ) } \\
\hline C-reactive protein $\left(\mathrm{mgl}^{-1}\right)$ & 1.27 & (1.03 to 1.57$)$ & 1.43 & $(1.20$ to 1.70$)$ \\
\hline Leptin $\left(\mathrm{ng} \mathrm{ml} \mathrm{l}^{-1}\right)$ & 1.10 & (0.98 to 1.34$)$ & 1.25 & (1.10 to 1.41$)$ \\
\hline Interleukin-6 (pg ml$\left.{ }^{-1}\right)$ & 1.16 & (1.06 to 1.33$)$ & 1.10 & (0.96 to 1.27$)$ \\
\hline
\end{tabular}

Abbreviations: $\mathrm{Cl}$, confidence interval; SII, slope index of inequality. ${ }^{\mathrm{a}}$ Additionally adjusted for height and height squared. $\mathrm{N}=7722$ participants with multivariate imputation. All analyses are adjusted for age. SII represents the mean (or geometric mean) difference between the individuals of lowest and highest socioeconomic position on the hypothetical underlying continuous distribution of maternal education.

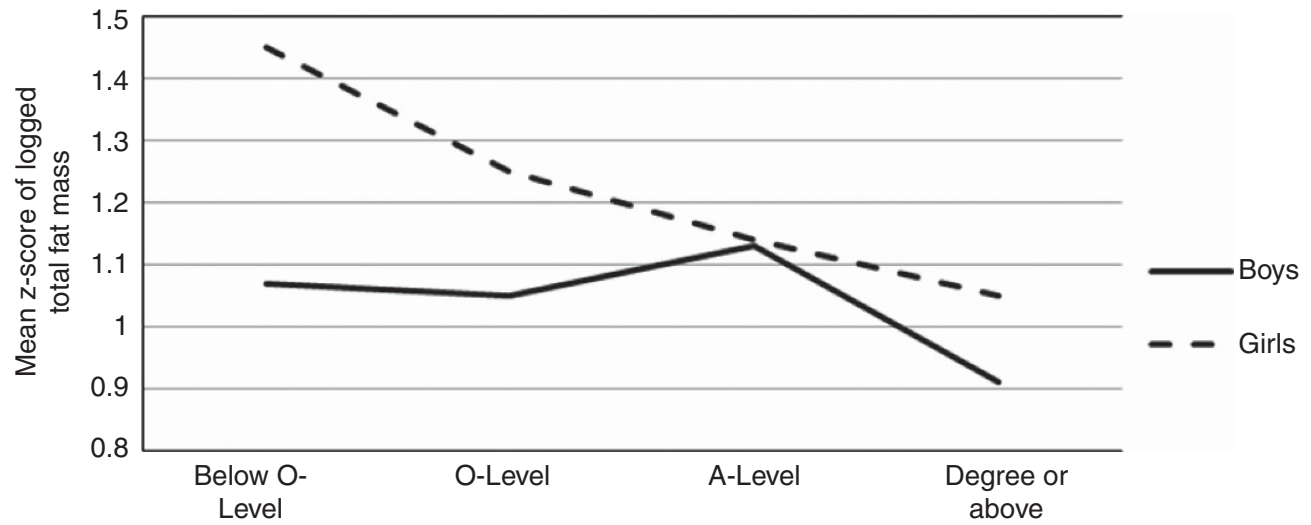

Figure 1 Mean levels of z-scores total fat mass across categories of maternal education for boys and girls.

non-missing data set). An increase in total fat mass was associated with a decrease in HDL, Apo B and adiponectin, and an increase in each of the other cardiovascular risk factors.

Mediation of socioeconomic inequalities in cardiovascular risk factors by adiposity

The inequalities in leptin and CRP were almost completely mediated by adiposity, because the coefficients of the total and indirect pathways are approximately the same size (Table 4). The degree to which inequalities in the other cardiovascular risk factors were mediated by adiposity was variable. For Apo B and IL-6, the coefficients for the indirect pathway (that is, mediated by adiposity) were approximately half to a third of the total effect size. For blood pressure, a lower proportion of the socioeconomic effect appears to be mediated by adiposity - the size of the coefficient for the indirect pathways is approximately half to a quarter of the total effect size in girls and a fifth to a sixth in boys.

Although no association was observed between maternal education and cholesterol, triglycerides, HDL, Apo A1 or adiponectin, there is evidence of an indirect pathway between maternal education and each of these outcomes through total fat mass. This is because each of these 


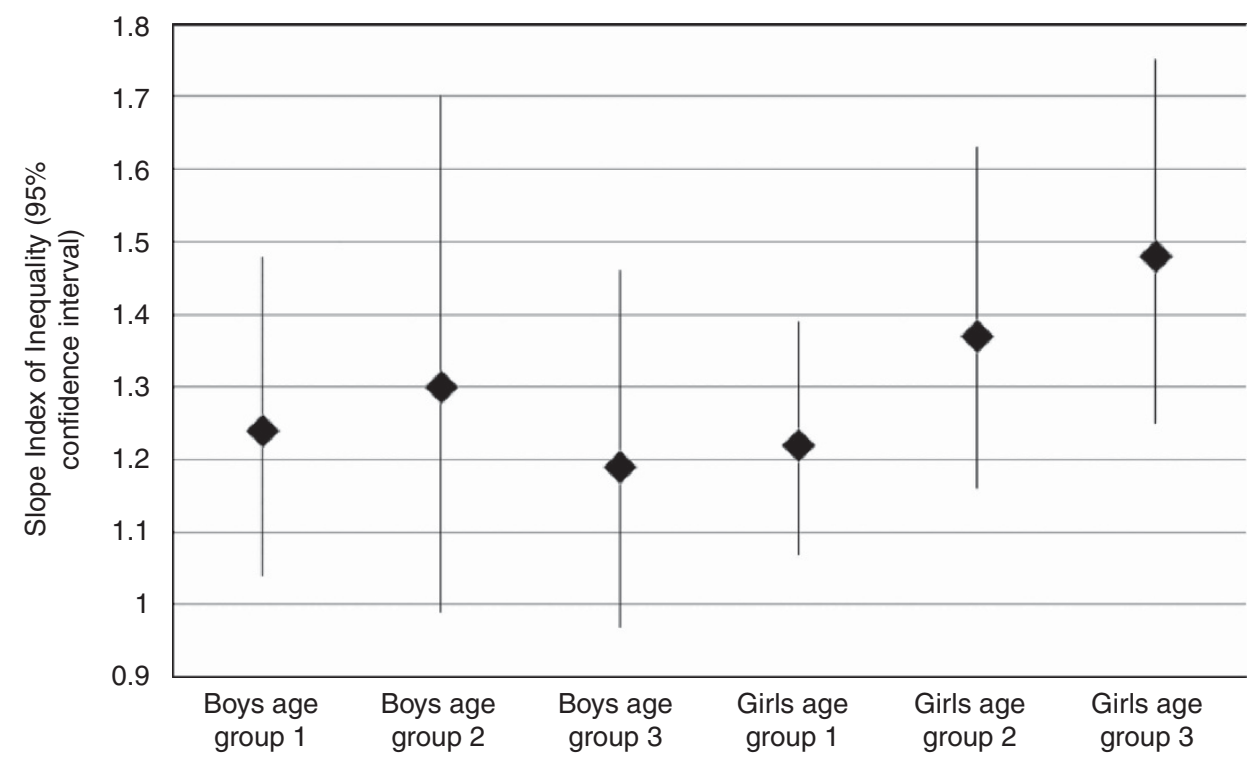

Figure 2 Slope index of inequality for DXA-assessed total fat mass, using maternal education as the SEP indicator, separately by tertiles of age and gender. Age ranges of tertiles were boys: 105-118 months, 118-120 months, 120-140 months, and girls: 106-118 months, 118-120 months, 120-140 months.

Table 3 Association between DXA-assessed total fat mass and cardiovascular risk factors at age 9.9 years

\begin{tabular}{|c|c|c|c|c|}
\hline & \multicolumn{2}{|c|}{ Boys, $\mathrm{N}=3809$} & \multicolumn{2}{|c|}{ Girls, $N=3913$} \\
\hline & $\beta$ & $95 \% \mathrm{Cl}$ & $\beta$ & $(95 \% \mathrm{Cl})$ \\
\hline Cholesterol $\left(\mathrm{mmoll}^{-1}\right)$ & 0.00002 & (0.00001 to 0.00002$)$ & 0.00002 & (0.00001 to 0.00002$)$ \\
\hline Triglycerides $\left(\mathrm{mmoll}^{-1}\right)$ & 0.00003 & (0.00002 to 0.00003$)$ & 0.00003 & (0.00002 to 0.00003$)$ \\
\hline High-density lipoprotein $\left(\mathrm{mmoll}^{-1}\right)$ & -0.00002 & $(-0.00002$ to -0.00001$)$ & -0.00002 & $(-0.00002$ to -0.00002$)$ \\
\hline Apolipoprotein A1 (mg per $100 \mathrm{ml}$ ) & -0.0006 & $(-0.0008$ to -0.0004$)$ & -0.0007 & $(-0.0009$ to -0.0005$)$ \\
\hline Apolipoprotein B (mg per $100 \mathrm{ml}$ ) & 0.0006 & $(0.0005$ to 0.0007$)$ & 0.0007 & $(0.0005$ to 0.0008$)$ \\
\hline Adiponectin $\left(\mathrm{mg} \mathrm{ml}^{-1}\right)$ & -0.09 & $(-0.14$ to -0.05$)$ & -0.17 & $(-0.21$ to -0.13$)$ \\
\hline Systolic blood pressure & 0.0007 & $(0.0006$ to 0.0007$)$ & 0.0007 & $(0.0006$ to 0.0008$)$ \\
\hline Diastolic blood pressure & 0.0003 & (0.0002 to 0.0003$)$ & 0.0003 & (0.0002 to 0.0003$)$ \\
\hline Logged C-reactive protein $\left(\mathrm{mgl}^{-1}\right)$ & 0.0001 & (0.00009 to 0.0001$)$ & 0.0001 & (0.0001 to 0.0001$)$ \\
\hline Logged leptin $\left(\mathrm{ng} \mathrm{ml}^{-1}\right)$ & 0.0001 & (0.0001 to 0.00013$)$ & 0.0001 & (0.0001 to 0.0001$)$ \\
\hline Logged interleukin-6 $\left(\mathrm{pg} \mathrm{ml}^{-1}\right)$ & 0.00004 & (0.00002 to 0.00005$)$ & 0.00004 & (0.00003 to 0.00005$)$ \\
\hline
\end{tabular}

Abbreviation: $\mathrm{Cl}$, confidence interval. $\mathrm{N}=7722$ participants with multivariate imputation. Linear regression coefficients from regressions of total fat mass on the cardiovascular risk factor, adjusted for age, height and height squared. Age, height and height squared were centred on mean values (for boys and girls separately), such that coefficients represent the change in the cardiovascular risk factor for a $1 \mathrm{~kg}$ increase in total fat mass at 9.9 years in a child of average height.

outcomes is associated with total fat mass, which itself is associated with maternal education.

\section{Discussion}

Inequalities in cardiovascular risk factors, and mediation by adiposity

Inequalities in some, although not all, cardiovascular risk factors were evident among these young children, with socioeconomic differentials generally being wider in girls than boys. For both boys and girls, weak associations were observed between maternal education and CRP, IL-6 and leptin, and stronger associations were observed for Apo B, systolic and diastolic blood pressure (inequalities in diastolic blood pressure were present only in girls).

The extent to which the inequalities in these cardiovascular risk factors were mediated by adiposity was variable. Almost the entire association between maternal education and leptin and CRP was mediated through fat mass. This is an expected result for leptin, given its very strong correlation with fat mass. For IL-6, Apo B, systolic and diastolic blood pressure there was evidence of mediation through adiposity, 
Table 4 Mediation of the association between maternal education and cardiovascular risk factors by DXA-assessed total fat mass: standardised direct and indirect effects of maternal education

\begin{tabular}{|c|c|c|c|c|}
\hline \multirow[t]{2}{*}{ Cardiovascular risk factor } & \multicolumn{2}{|c|}{ Boys, $\mathrm{N}=3809$} & \multicolumn{2}{|c|}{ Girls, $N=3913$} \\
\hline & Total effect $(95 \% \mathrm{Cl})^{\mathrm{a}}$ & Indirect effect $(95 \% \mathrm{CI})^{\mathrm{b}}$ & Total effect $(95 \% \mathrm{Cl})^{\mathrm{a}}$ & Indirect effect $(95 \% \mathrm{Cl})^{\mathrm{b}}$ \\
\hline Cholesterol $\left(\mathrm{mmoll}^{-1}\right)$ & $0.030(-0.10$ to 0.070$)$ & 0.009 (0.004 to 0.015$)$ & $0.030(-0.012$ to 0.073$)$ & $0.014(0.008$ to 0.020$)$ \\
\hline Triglycerides $\left(\mathrm{mmoll}^{-1}\right)$ & $0.004(-0.036$ to 0.044$)$ & 0.011 (0.005 to 0.017$)$ & $0.014(-0.027$ to 0.055$)$ & $0.026(0.017$ to 0.034$)$ \\
\hline High-density lipoprotein $\left(\mathrm{mmoll}^{-1}\right)$ & $0.012(-0.028$ to 0.052$)$ & $-0.014(-0.022$ to -0.007$)$ & $-0.031(-0.071$ to 0.009$)$ & $-0.030(-0.039$ to -0.020$)$ \\
\hline Apolipoprotein A1 (mg per $100 \mathrm{ml}$ ) & $0.033(-0.007$ to 0.073$)$ & $-0.007(-0.011$ to -0.002$)$ & $-0.001(-0.041$ to 0.039$)$ & $-0.018(-0.025$ to -0.011$)$ \\
\hline Apolipoprotein B (mg per $100 \mathrm{ml}$ ) & $0.042(0.002$ to 0.082$)$ & $0.015(0.007$ to 0.023$)$ & $0.063(0.022$ to 0.10$)$ & $0.025(0.016$ to 0.033$)$ \\
\hline Adiponectin $\left(\mathrm{mg} \mathrm{ml}^{-1}\right)$ & $0.022(-0.018$ to 0.062$)$ & $-0.001(-0.004$ to 0.002$)$ & $-0.022(-0.062$ to 0.019$)$ & $-0.016(-0.023$ to -0.010$)$ \\
\hline Systolic blood pressure & $0.10(0.068$ to 0.13$)$ & 0.021 (0.010 to 0.031$)$ & 0.095 (0.064 to 0.13$)$ & 0.037 (0.026 to 0.049$)$ \\
\hline Diastolic blood pressure & $0.069(0.036$ to 0.10$)$ & 0.012 (0.006 to 0.019$)$ & $0.083(0.050$ to 0.12$)$ & 0.022 (0.015 to 0.029$)$ \\
\hline Logged C-reactive protein $\left(\mathrm{mg} \mathrm{l}^{-1}\right)^{\mathrm{a}}$ & $0.031(-0.008$ to 0.070$)$ & $0.028(0.014$ to 0.041$)$ & $0.063(0.024$ to 0.10$)$ & $0.052(0.037$ to 0.068$)$ \\
\hline Logged leptin $\left(\mathrm{ng} \mathrm{ml}^{-1}\right)^{\mathrm{a}}$ & 0.047 (0.012 to 0.082$)$ & $0.051(0.026$ to 0.076$)$ & $0.086(0.052$ to 0.12$)$ & $0.086(0.060$ to 0.11$)$ \\
\hline Logged interleukin-6 $\left(\mathrm{pg} \mathrm{ml}^{-1}\right)^{\mathrm{a}}$ & $0.043(0.004$ to 0.083$)$ & $0.13(0.006$ to 0.020$)$ & $0.046(0.005$ to 0.086$)$ & $0.026(0.017$ to 0.035$)$ \\
\hline
\end{tabular}

Abbreviation: $\mathrm{Cl}$, confidence interval. ${ }^{\mathrm{a}}$ Total effect: the total effect of maternal education on the cardiovascular risk factor. ${ }^{\mathrm{b}}$ Indirect effect: the effect of maternal education on the cardiovascular risk factor that is mediated through DXA-assessed total fat mass. $N=7722$ participants with multivariate imputation. All analyses adjusted for age, height and height squared. Results are standardised coefficients from simultaneous linear regressions in path analysis, so they estimate the change in s.d. of cardiovascular risk factor associated with a one s.d. increase in maternal education.

but the proportion of the inequalities mediated through adiposity was lower.

Although a gradient of higher levels of adult obesity with decreasing SEP has long been established in high-income countries, ${ }^{34}$ a similar socioeconomic gradient in childhood adiposity is a relatively recent phenomenon. In the past, disadvantaged groups would have been more likely to be less adipose rather than suffering from a higher burden of obesity. A review of cohorts published in 2002 (children aged up to 18 and young adults aged up to 24, who had largely been born between the 1940s and 1980s, although some studies of children born in the early 1990s were included) did not show socioeconomic inequalities in adiposity in childhood, but did show some evidence of socioeconomic patterning of adiposity in young adults. ${ }^{12}$ Other cardiovascular risk factors, including two that showed a socioeconomic gradient in this study (blood pressure and CRP), were also not found to be socially patterned in children and younger adults in previous generations. ${ }^{12}$ In a study of Glasgow students (attending the University Health Service between 1948 and 1968), lower childhood parental occupational social class did not predict early adult adiposity (mean age 23 years in men and 20 years in women), but did predict later adult BMI (mean age 39 years in men and 36 years in women), in spite of the little heterogeneity in their adult SEP. ${ }^{35}$ This lack of social gradient in early life adiposity among cohorts of today's adults, together with the fact that relatively few individuals would have been overweight or obese in childhood in these cohorts, implies that childhood obesity may not be a major contributor to the socioeconomic gradients in adult cardiovascular disease observed in many studies of contemporary adults who were born during the 1940 s or earlier. ${ }^{36}$ However, the socioeconomic patterning of adiposity and other cardiohyphen-qj; vascular risk factors we observe in these contem- porary children suggests that socioeconomic gradients in adult adiposity, and also therefore in cardiovascular disease and particularly diabetes, may widen as today's children become adults. Interventions targeting inequalities in childhood adiposity may therefore be an important aspect of prevention of widening diabetes and cardiovascular disease inequalities in the future when these children become adults.

As with adiposity, blood pressure tends to be higher among lower socioeconomic groups in current adults. ${ }^{37,38}$ Social patterning of blood pressure was not observed in children among previous generations. ${ }^{12,39}$ We did see a socioeconomic gradient in blood pressure in the children in this study, implying that inequalities in hypertension may widen as these children become adults. It has been shown in this population that blood pressure is associated with both fat and lean mass. ${ }^{40}$ For diastolic blood pressure in both boys and girls, and additionally for systolic blood pressure in boys, a large proportion of the association with SEP in our analyses was not mediated by adiposity. This suggests that factors other than adiposity are contributing to socioeconomic inequalities in blood pressure, even in these young children. These factors could possibly include dietary patterns, physical activity or other potentially modifiable risk factors for high blood pressure, but require further exploration.

ApoB was more strongly socially patterned in this population than plasma cholesterol. Previous research has suggested that ApoB is more strongly related to insulin resistance than cholesterol and related risk factors (for example, triglycerides, CRP, and so on). ${ }^{41}$ It has also been shown to be potentially more predictive of fatal myocardial infarction than cholesterol. ${ }^{42}$ The association with ApoB, as with other risk factors was, however, largely mediated through socioeconomic inequalities in adiposity. 
Gender differences in inequalities in adiposity

We observed socioeconomic inequality in adiposity in both boys and girls, but the disparities were wider among girls. Among girls, there was a clear gradient of decreasing total fat mass across all categories of increasing maternal education. Among boys, in contrast, adiposity was lower among boys whose mothers had the highest educational level, but similar across the lower categories of maternal education.

The association between SEP and adiposity is known to be generally stronger in adult women than men, ${ }^{34}$ but gender differences are less consistent in contemporary children. In a systematic review of socioeconomic status and adiposity in school-aged children using studies from Western developed countries published since 1989, over half of the 19 studies reporting associations separately for boys and girls showed no gender difference in the association. Findings from the remaining studies were inconsistent. ${ }^{6}$ Contemporary children may be shifting towards the social patterning observed in adults, implying again that inequalities may be widening over time and with this becoming more prominent in females. Gender differences in the social patterning of adiposity are largely unexplained. It may, at least in part, be because of gender differences in the social patterning of physical activity. There is some evidence that boys are more likely to participate in sport than girls, regardless of their $\mathrm{SEP}^{43}$ and that the inverse association between physical activity and adiposity tends to be stronger and more common among boys than girls. ${ }^{44,45}$ Within ALSPAC, there is little evidence of a socioeconomic gradient in physical activity, ${ }^{46}$ but there is evidence that boys are more likely to participate in moderate to vigorous activity, ${ }^{46}$ which has been shown to be more important than total activity for preventing obesity. ${ }^{45}$ Furthermore, women of higher SEP may be more susceptible to pressure to stay slim, and children may be becoming exposed to such pressures at this early age.

It is possible, however, that the weaker associations between SEP and adiposity and cardiovascular risk factors observed in boys in our study simply reflects girls at this age being biologically older than boys, that is, closer to puberty. The vast majority of both boys and girls were pre-pubertal in this cohort according to their Tanner stage (Table 1), but this relatively crude measure cannot give a specific indication of biological age. The hypothesis that the gender differences observed in this study may be at least partially driven by girls being biologically older is supported by the fact that we saw clear age-gradients in the social patterning of obesity within girls, with older girls showing greater socioeconomic differentials than younger girls, but less clear age patterning of inequality among boys.

\section{Study strengths and limitations}

A major strength of this study is that it is the largest study to date to report socioeconomic inequalities in a wide range of cardiovascular risk factors in a cohort of contemporary children, and to examine the extent to which these inequalities are mediated by adiposity using DXA-assessed total fat mass.

We used path analysis to determine mediation of inequalities in cardiovascular risk factors by adiposity. This permits estimation of the proportion of the effect of SEP that is mediated through adiposity. We adjusted analyses for potential confounders (age, height and height squared) but the interpretation of the results from path analysis as causal estimates rests on the assumption of no unmeasured or residual confounding, similar to the use of standard regression methods.

Participation in most research studies is socially patterned, and ALSPAC is no exception. Those attending the follow-up clinic tended to be of higher SEP than the full cohort. This may have resulted in an underestimation of the inequalities in cardiovascular risk factors. There was minimal missing data among clinic participants for most variables but approximately one-third had data missing on blood assay risk factors. However, the results based on analysis of the non-missing data set and analyses using multivariate multiple imputation were similar and the similar patterning of associations with adiposity (for which there was very little missing data) and blood assay results, suggest that missing data has not resulted in major bias.

Offspring blood tests were completed on non-fasting blood samples but the majority of measures are not appreciably altered by this approach. ${ }^{47-49}$ Non-fasting triglyceride is at least as strongly linked to vascular outcomes as is fasting triglyceride, ${ }^{50}$ suggesting that for triglycerides also fasting status may not result in important biases with associations.

\section{Implications}

Our work shows important socioeconomic inequalities in adiposity and associated cardiovascular risk factors, particularly in girls, in a contemporary UK population of 10 -yearold children. Such childhood associations were not present in previous generations. Our results suggest that inequalities in adiposity, cardiovascular disease and diabetes may widen over time as these cohorts of children become adults. These findings highlight the importance of interventions aimed at preventing obesity in childhood. Population-based interventions, if successful, may have a greater overall population effect on the reduction of obesity and its associated complications than interventions targeted at lower socioeconomic groups. However, it is well known that many interventions are initially taken up to a greater extent by more socially advantaged groups, leading to a widening of socioeconomic differentials. To reduce socioeconomic inequalities in obesity, cardiovascular disease and diabetes, it will be necessary to either target interventions at disadvantaged groups, or to attempt to promote their participation in population-wide interventions. 


\section{Conflict of interest}

The authors declare no conflict of interest.

\section{Acknowledgements}

We are extremely grateful to all the families who took part in this study, the midwives for their help in recruiting them, and the whole ALSPAC team, which includes interviewers, computer and laboratory technicians, clerical workers, research scientists, volunteers, managers, receptionists and nurses. The UK Medical Research Council, the Wellcome Trust and the University of Bristol provide core support for ALSPAC. This publication is the work of the authors and Laura Howe will serve as guarantor for the contents of this paper. The UK Medical Research Council and the University of Bristol provide core funding for the MRC Centre of Causal Analyses in Translational Epidemiology. This work was funded by a grant from the UK Economic and Social Research Council (RES-060-23-0011). This grant provides the salary for LH. BG is funded by a UK Medical Research Council Fellowship in Health of the Public.

\section{References}

1 Galobardes B, Lynch JW, Davey Smith G. Childhood socioeconomic circumstances and cause-specific mortality in adulthood: systematic review and interpretation. Epidemiol Rev 2004; 26: 7-21.

2 Galobardes B, Davey Smith G, Lynch JW. Systematic review of the influence of childhood socioeconomic circumstances on risk for cardiovascular disease in adulthood. Ann Epidemiol 2006; 16: 91-104.

3 Galobardes B, Lynch JW, Davey Smith G. Is the association between childhood socioeconomic circumstances and causespecific mortality established? Update of a systematic review. J Epidemiol Community Health 2008; 62: 387-390.

4 Agardh EE, Ahlbom A, Andersson T, Efendic S, Grill V, Hallqvist J et al. Explanations of socioeconomic differences in excess risk of type 2 diabetes in swedish men and women. Diabetes Care 2004; 27: 716-721.

5 Evans JMM, Newton RW, Ruta DA, MacDonald TM, Morris AD. Socio-economic status, obesity and prevalence of type 1 and type 2 diabetes mellitus. Diabet Med 2000; 17: 478-480.

6 Shrewsbury V, Wardle J. Socioeconomic status and adiposity in childhood: a systematic review of cross-sectional studies 1990-2005. Obesity 2008; 16: 275-284.

7 Owen CG, Whincup PH, Orfei L, Chou Q-A, Rudnicka AR, Wathern AK et al. Is body mass index before middle age related to coronary heart disease risk in later life? Evidence from observational studies. Int J Obes 2009; 33: 866-877.

8 Davey Smith G, Hart C. Insulin resistance syndrome and childhood social conditions. Lancet 1997; 349: 284-285.

9 Lawlor DA, Ebrahim S, Davey Smith G. Socioeconomic position in childhood and adulthood and insulin resistance: cross sectional survey using data from the British women's heart and health study. Br Med J 2002; 325: 805-807.

10 Lawlor DA, Harro M, Wedderkopp N, Andersen LB, Sardinha LB, Riddoch CJ et al. The association of socioeconomic position with insulin resistance among children from northern (Denmark), eastern (Estonia) and southern (Portugal) Europe: findings from the European youth heart study. Br Med J 2005; 331: 183-186.

11 Loucks EB, Magnusson KT, Cook S, Rehkopf DH, Ford ES, Berkman LF. Socioeconomic position and the metabolic syndrome in early, middle, and late life: evidence from NHANES 1999-2002. Ann Epidemiol 2007; 17: 782-790.

12 Batty GD, Leon DA. Socio-economic position and coronary heart disease risk factors in children and young people. Eur J Public Health 2002; 12: 263-272.

13 van Lenthe FJ, Boreham CA, Twisk JW, Strain JJ, Savage JM, Davey Smith G. Socio-economic position and coronary heart disease risk factors in youth. Findings from the young hearts project in Northern Ireland. Eur J Public Health 2001; 11: 43-50.

14 Ness AR, Leary S, Reilly J, Wells J, Tobias J, Clark E et al. The social patterning of fat and lean mass in a contemporary cohort of children. Int J Pediatr Obes 2006; 1: 59-61.

15 Golding J, Pembrey M, Jones R, the ALSPAC Study Team. ALSPAC - The avon longitudinal study of parents and children I. Study methodology. Paediatr Perinatal Epidemiol 2001; 15: 74-87.

16 Morris NM, Udry JR. Validation of a self-administered instrument to assess stage of adolescent development. J Youth Adolesc 1980; 9: 271-280.

17 Duke PM, Litt IF, Gross RT. Adolescents' self-assessment of sexual maturation. Pediatrics 1980; 66: 918-920.

18 Myers GL, Kimberly MM, Waymack PP, Smith SJ, Cooper GR, Sampson EJ. A reference method laboratory network for cholesterol: a model for standardization and improvement of clinical laboratory measurements. Clin Chem 2000; 46: 1762-1772.

19 Wallace AM, McMahon AD, Packard CJ, Kelly A, Shepherd J, Gaw A et al. Plasma leptin and the risk of cardiovascular disease in the west of Scotland coronary prevention study (WOSCOPS). Circulation 2001; 104: 3052-3056.

20 Sergeant JC, Firth D. Relative index of inequality: definition, estimation, and inference. Biostatistics 2006; 7: 213-224.

21 Kraemer HC, Stice E, Kazdin A, Offord D, Kupfer D. How do risk factors work together? Mediators, moderators, and independent, overlapping, and proxy risk factors. Am J Psychiatry 2001; 158: 848-856.

22 Hafeman DM, Schwarz S. Opening the black box: a motivation for the assessment of mediation. Int J Epidemiol 2009; 38: 838-845.

23 Avenell A, Brown TJ, McGee MA, Campbell MK, Grant AM, Broom J et al. What are the long-term benefits of weight reducing diets in adults? A systematic review of randomized controlled trials. J Hum Nutr Diet 2004; 17: 317-335.

24 O'Meara S, Riemsma R, Shirran L, Mather L, ter Riet G. A systematic review of the clinical effectiveness of orlistat used for the management of obesity. Obes Rev 2004; 5: 51-68.

25 Chanoine JP, Hampl S, Jensen C, Boldrin M, Hauptman J. Effect of orlistat on weight and body composition in obese adolescents. J Am Med Assoc 2005; 23: 2873-2883.

26 Daniels SR, Long B, Crow S, Styne D, Sothern M, VargasRodirguez I et al. Cardiovascular effects of sibutramine in the treatment of obese adolescents: results of a randomized, doubleblind, placebo-controlled study. Pediatrics 2007; 120: e147-e157.

27 Freathy RM, Timpson NJ, Lawlor DA, Pouta A, Ben-Shlomo Y, Ruokonen A et al. Common variation in the FTO gene alters diabetes-related metabolic traits to the extent expected given its effect on BMI. Diabetes 2008; 57: 1419-1426.

28 Timpson NJ, Harbord R, Davey Smith G, Zacho J, TybjaergHansen A, Nordestgaard BG. Does greater adiposity increase blood pressure and hypertension risk? Mendelian randomization using the FTO/MC4R genotype. Hypertension 2009; 54: 84-90.

29 Baron RM, Kenny DA. The Moderator-mediator variable distinction in social psychological research: conceptual, strategic, and statistical considerations. J Pers Soc Psychol 1986; 51: 1173-1182.

30 Wright S. The method of path coefficients. Ann Math Stat 1934; 5: $161-215$. 
31 Schumacker RE, Lomax RG. A beginner's guide to structural equation modeling. Lawrence Erlbaum Associates; Mawhah, NJ, 2004.

32 http://www.statmodel.com/index.shtml [computer program]. 2007.

33 Royston P. Multiple imputation of missing values. Stata J 2004; 4: 227-241.

34 McClaren L. Socioeconomic status and obesity. Epidemiol Rev 2007; 29: 29-48.

35 Okasha M, McCarron P, McEwan J, Davey Smith G. Childhood social class and adulthood obesity: findings from the Glasgow alumni cohort. J Epidemiol Community Health 2003; 57: 508-509.

36 Davey Smith G, McCarron P, Okasha M, McEwen J. Social circumstances in childhood and cardiovascular disease mortality: prospective observational study of Glasgow university students. J Epidemiol Community Health 2001; 55: 340-341. economic status, blood pressure progression, and incident hypertension in a prospective cohort of female health professionals. Eur Heart J 2009; 30: 1305-1306.

38 Grotto I, Huerta M, Sharabi Y. Hypertension and socioeconomic status. Curr Opin Cardiol 2008; 23: 335-339.

39 Colhoun HM, Hemingway H, Poulter NR. Socio-economic status and blood pressure: an overview analysis. J Hum Hypertens 1998; 12: 91-110.

40 Brion MA, Ness AR, Davey Smith G, Leary SD. Association between body composition and blood pressure in a contemporary cohort of 9-year-old children. J Hum Hypertens 2007; 21: 283-290.

41 Sattar N, Williams K, Sniderman AD, D’Agostino R, Haffner SM. Comparison of the associations of apolipoprotein $\mathrm{B}$ and nonhigh-density lipoprotein cholesterol with other cardiovascular risk factors in patients with the metabolic syndrome in the insulin resistance atherosclerosis study. Circulation 2004; 110: 2687-2693.

42 Walldius G, Jungner I, Holme I, Aastveit AH, Kolar W, Steiner E. High apolipoprotein B, low apolipoprotein A-I, and improvement
37 Conen D, Glynn RJ, Ridker PM, Buring JE, Albert MA. Socio-

in the prediction of fatal myocardial infarction (AMORIS study): a prospective study. Lancet 2001; 358: 2026-2033.

43 Fairclough SJ, Boddy LM, Hackett AF, Stratton G. Associations between children's socioeconomic status, weight status, and sex, with screen-based sedentary behaviours and sport participation. Int J Pediatr Obes 2009; 4: 299-305.

44 Jimenez-Pavon D, Kelly J, Reilly J. Associations between objectively measured habitual physical activity and adiposity in children and adolescents: systematic review. Int I Pediatr Obes 2009 (e-pub ahead of print).

45 Ness AR, Leary SD, Mattocks C, Blair SN, Reilly JJ, Wells J et al. Objectively measured physical activity and fat mass in a large cohort of children. Plos Med 2007; 4: e97.

46 Riddoch CJ, Mattocks C, Deere K, Saunders J, Kirkby J, Tilling K et al. Objective measurement of levels and patterns of physical activity. Arch Dis Child 2007; 92: 963-969.

47 Rifai N, Merrill JR, Holly RG. Postprandial effect of a high fat meal on plasma lipid, lipoprotein cholesterol and apolipoprotein measurements. Ann Clin Biochem 1990; 27: 489-493.

48 Shand B, Elder P, Scott R, Frampton C, Willis J. Biovariability of plasma adiponectin. Clin Chem Lab Med 2006; 44: 1264-1268.

49 Poppitt SD, Keogh GF, Lithander FE, Wang Y, Mulvey TB, Chan YK et al. Postprandial response of adiponectin, interleukin-6, tumor necrosis factor-alpha, and C-reactive protein to a high-fat dietary load. Nutrition 2008; 24: 322-329.

50 Emerging Risk Factors Collaboration. Di Angelantonio E, Sarwar N, Perry P, Kaptoge S, Ray KK et al. Major lipids, apolipoproteins, and risk of vascular disease. J Am Med Assoc 2009; 302: 1993-2000.

(c) This work is licensed under a Creative
Commons Attribution 3.0 Unported Li-
cense. To view a copy of this license, visit http://
creativecommons.org/licenses/by/3.0/

Supplementary Information accompanies the paper on International Journal of Obesity website (http://www.nature.com/ijo) 